\title{
Acupuncture for Non-Verbal Autistic Children: Randomized Controlled Trial
}

\author{
Wen-Xiong Chen ${ }^{1, \dagger}$, Gang Liu ${ }^{2}$, Hong-Sheng Liư ${ }^{3}$, Zhi-Fang Huang' ${ }^{1}$ Si-Yuan Yang ${ }^{1}$, Ju Huang ${ }^{2}$, Si-Hui Zeng ${ }^{3}$, Jing-Yu
} Huang ${ }^{1}$, Qian-Qian $\mathrm{Wu}^{3}$

\begin{abstract}
\section{Objective}

To study the efficacy, safety, and the alterations of brain neurochemical of short-term acupuncture for children with non-verbal autism spectrum disorder.
\end{abstract}

\section{Methods}

Study design: randomized, simple-blind, controlled trial. Non-verbal autistic children were randomly assigned to acupuncture group $(n=24)$ or control group $(n=19)$. The acupuncture group received 20 sessions for two acupoints on head [Temporal Anterior Oblique (MS6) under 2/5 and Temporal Posterior Oblique (MS7) under 2/5] over 4 weeks. Primary outcome measures comprised of Autism Treatment Evaluation Checklist (ATEC), Reynell Developmental Language Scale (RDLS), Pediatric Evaluation Disability Inventory (PEDI) and Clinical Global Impression-Improvement (CGI-I) scale. Secondary outcome measures included Gesell Development Diagnosis Scale (GDDS)/ Wechsler Preschool and Primary Scale of Intelligence-Fourth edition (WPPSI-IV)/Wechsler Intelligence Scale for Children-Fourth edition (WISC-IV), brain Magnetic Resonance Spectroscopy (MRS) and Weekly Parental Report.

\section{Results}

There were significant ameliorations in the self-care $(p=0.045)$ and self-care assistant $(p=0.019)$ domains of PEDI, and CGI-I $(p=0.003)$ in the acupuncture group, in contrast to control group. The significantly better social initiation $(P=0.003)$, receptive language $(p=0.002)$, pointing $(p=0.036)$, imitation ( $p=0.025)$, and motor skills $(p=0.036)$ were also revealed, regarding the Weekly Parental Report. As for the brain MRS, the acupuncture group showed significantly decreased the ratio of Choline /Creatine ( $p=0.037)$ at the left grey matter of Inferior frontal gurus and increased the concentrations of Creatine $(p=0.036)$ at the right grey matter of superior temporal gurus. Mild side effects of irritability at initial sessions during acupuncture were found.

\section{Conclusion}

A short-term intensive acupuncture for selected points is helpful to improve specific functions of non-verbal autistic children, especially for self-care ability, which may be due to regulating cellular renewal and energy metabolism after acupuncture.

'Department of Neurology, Brain Center, Guangzhou Women and Children's Medical Center, Guangzhou Medical University, Guangzhou City, Guangdong Province, China

2Department of Rehabilitation, the Third Affiliated Hospital of Southern Medical University, Guangzhou City, Guangdong Province, China

${ }^{3}$ Department of Radiology, Guangzhou Women and Children's Medical Center, Guangzhou Medical University, Guangzhou City, Guangdong Province, China

${ }^{\dagger}$ Author for correspondence: Dr. Wen-Xiong Chen, Department of Neurology, Brain Center, Guangzhou Women and Children's Medical Center, Guangzhou Medical University, 9\# Jin Sui Road, 510623, Guangzhou City, Guangdong Province, P.R. of China, Tel: 86-02038076127; email: gzchcwx@126.com 


\section{Keywords}

Autism Spectrum Disorder (ASD), Acupuncture, Non-verbal, Children, Magnetic Resonance Spectroscopy (MRS), Randomized controlled trial (RCT)

\section{Introduction}

Autism spectrum disorder (ASD) is a neurodevelopmental disorder, characterized by the dyad of deficits of social relatedness and communication, and stereotypic behaviors [1]. Language proficiency being one of most significant predicting factors regarding the prognosis of ASD was stated [2]. About one fourth of patients with ASD retained non-verbal status [3]. The severe speech impairment is one of the most difficult to treat characteristics of children with ASD [4]. Helping autistic children acquiring language has been very concerned in the ASD intervention [5].

The disturbances of mirror neuron system (MNS) and its relevant networks are thought to be associated with social, communication, as well as emotional regulation of ASD [6]. The Broca area at the inferior frontal gurus [7], are strongly related to human language, containing MNS [8], which touch upon the perception and apprehension of human activities, also participate a higher level of cognitive processing e.g. language or imitation [9]. Other areas, including the superior temporal sulcus and the top of inferior parietal, are also considered to consist of MNS [10].

There is no enough evidence or consensus regarding the best interventions for the key features of ASD [11]. The devise of a therapy method to the involvement in the MNS mechanism may serve as an important clinical application potential [12]. Acupuncture has been extensively performed in China and is being increasingly carried out in more and more Western countries. It is hypothesized that acupuncture may serves as an ideal intervention pattern to be engagement with the MNS mechanism, via its corresponding acupoints.

Proton magnetic resonance spectroscopy (1H-MRS) is a brain imaging technique with the permission of non-invasive quantification of neurochemicals in the endogenous brain and examination of regional cellular activity and function in the living subjects.

Therefore, we performed randomized controlled trial of acupuncture for non-verbal autistic children to study the efficacy, safety, and also explore the changes of brain neurochemicals via $1 \mathrm{H}-\mathrm{MRS}$. This is the first simple-blind, randomized controlled trial of acupuncture for non-verbal autistic children and is according to the selected acupoints from our previous small case series [13]. This trial was registered at Chinese ClinicalTrial.gov (2017 Feb 07; identifier: ChiCTR-IPR-17010558).

\section{Material and Methods}

The current randomized controlled trial was performed from Feb 2017 to June 2018 at Guangzhou Women and Children's Medical Center, Guangzhou Medical University, with the senior author (WX Chen) as principal investigator, co-cooperation with the Department of Rehabilitation, the Third Affiliated Hospitals of Southern Medical University. The research protocol was approved by the Institutional Review Board of the Guangzhou Women and Children's Medical Center. Written informed consents were obtained from the parents/guardians.

Participants were enlisted from a neuropsychological clinic specializing in ASD. In addition, the recruit advertisement was published in the local newspapers to invite the parents with non-verbal autistic children to participate. The clinical history and comprehensive physical / neurological examination along with relevant assessments were performed during the respondent's initial interview. The evaluations of spontaneously using functional speech were obtained through a brief interview with the parent/caregiver, also by the clinical observation during the comprehensive assessments.

\section{- Inclusion criteria}

Children with ASD meeting the following criteria were included:

1. Diagnostic and Statistical Manual of Mental Disorders ( ( $^{\text {th }}$ Edition) (DSM-5) [1]

2. Autism Diagnostic Interview-Revised (ADI-R) [14]

3. Autism Diagnostic Observation Schedule (ADOS) [15]

4. Definition of non-verbal status: Spontaneous use of functional words less than five

5. Age between 1.5 and 14 years 
6. Have not accepted the acupuncture intervention before

\section{n Exclusion criteria}

Exclusion criteria included those patients associated with neurologic disorders such as epilepsy.

The eligibility of the included subjects was confirmed by the principal investigator (Chen WX). The wash-out period for included subjects lasted two weeks to provide a psychological preparation for parents/guardians and to hold back other possible confounding factors.

\section{- Randomization and concealment allocation}

Randomized assignment procedure was stratifiedily performed. Generated randomization numbers were generated by a computer program, matched by chronological age and severity of autism, use of Childhood Autism Rating Scale (CARS) [16], being a diagnostic tool designed to evaluate severity of autism in children with ASD. Allocation of each child was randomly carried out by drawing an envelope which contained a randomization number.

The assessor and the investigator of brain MRS were blinded to groupings' allocation. The parent/guardian and acupuncturist were not blinded to group allocations.

\section{- Intervention}

The acupuncture was practiced by the same acupuncturist. The children in both groups could continue special education if he/she had already been received it during the acupuncture.

\section{- Acupuncture group}

Two acupoints [the temporal anterior oblique (MS6) under 2/5 and temporal posterior oblique (MS7) under 2/5] on bilateral head were chosen, and alternately adopted for acupuncture [13].

Acupuncture contained 20 sessions, with consecutive 5 sessions from Monday to Friday per week, each session lasting 30 minutes, spanned over 4 weeks. A sterile disposable $0.3 \times 4 \mathrm{~cm}$ acupuncture needle (made in SuZhou, China, HWA-TO) was adopted. No sedation was needed.

During acupuncture, the subject was required to be in a sitting position when acupunctured, but could freedom walk after acupuncture.

\section{- Control group}

The subjects in the control group did not accept any acupuncture intervention.

\section{- Outcome measures}

The primary outcome measures included Autism Treatment Evaluation Checklist (ATEC), Pediatric Evaluation Disability Inventory (PEDI), Reynell Developmental Language Scale (RDLS), and Clinical Global Impression-Improvement (CGI-I) Scale. Secondary outcome measures consisted of Gesell Developmental Diagnostic Scale (GDDS)/The Wechsler Preschool and Primary Scale of Intelligence-Fourth edition (WPPSI-IV)/Wechsler Intelligence Scale for Children-Fourth edition (WISC-IV), brain Magnetic Resonance Spectroscopy (MRS) and Weekly Parental Report.

\section{- Parental assessment}

The following outcome measures were finished by parents/guardians. The parents/guardians were taught on how to properly use the following assessment tools during the baseline assessment.

Autism Treatment Evaluation Checklist (ATEC) [17]: A scale for rating symptoms of patients with ASD before and after treatment, containing five subscales of Speech/Language/ Communication, Sociability, Sensory/Cognitive Awareness, Health/Physical/Behavior, and Total ATEC Summary Score, adopted at baseline and post-treatment.

Pediatric Evaluation Disability Inventory (PEDI) [18]: A gauge of functional ability in children, in consideration of the use of special equipment and amount of caregiver assistance, containing 197 functional skills items, 20 caregiver assistance activities, and 20 environmental modifications, adopted at baseline and post-treatment.

Clinical Global Impression-Improvement (CGI-I) Scale [19]: The Clinical Global Impression-Improvement (CGI-I) scale is a seven-point scale requiring the assessor to assess how much the subject's illness has improved or worsened in term of a baseline state. Subjects were graded on a Likert scale of $1-7$, with $1=$ very much improved, $2=$ much improved, 3=minimally improved, $4=$ no change, $5=$ minimally worse, $6=$ much worse, and $7=$ very much worse, adopted at posttreatment.

Weekly parental report: A self-devised report was adopted for parents to record weekly changes during acupuncture containing the opening questions to answer in a written pattern accordingly. 


\section{- Assessor assessment}

The following outcome measures were carried out at baseline and post-treatment by the same assessor.

Gesell Developmental Diagnostic Scale (GDDS) [20]: A gauge of developmental quotient (DQ) in the following domains, consisted of gross motor, fine motor, adaptability, language, and personal-social behavior.

Wechsler Preschool and Primary Scale of Intelligence (WPPSI-IV) [21]: The WPPSIIV is an individually administered IQ test used with children aged from 2 years and 6 months to 7 years and 7 months. There are 14 different subtests on the WPPSI-IV.

Wechsler Intelligence Scale for Children (WISCIV) [22]: The WISC-IV is an individually administered Intelligence Quotient (IQ) test used with children aged from 6 to 16 years. There are 15 subtests on the WISC-IV.

Reynell Developmental Language Scale (RDLS) [23]: A gauge of a child's abilities on receptive and expressive language.

\section{- Brain magnetic resonance spectroscopy (MRS) assessment}

The SIEMENS 3.0T Skyra MR scanner with short TE multil-voxel ${ }^{1} \mathrm{H}$ MRS was used in current study.

\section{- MRS scan parameters}

The $1500 \mathrm{~ms}$ of TR and $40 \mathrm{~ms}$ of TE with the voxel dimensions being $40 \mathrm{~mm} \times 60 \mathrm{~mm}$ at the inferior frontal gurus (IFG) and superior temporal gurus (STG), and 90deg of Flip angle were adopted.

\section{- Data acquisition}

The bilateral gray matters of IFG, STG, cerebellum and hippocampus were selected to display the compounds including NAA, Cr, Cho, Ins, $\mathrm{Cr} 2$ and Lac. The examples of acquisition of relevant MRS line and compound ratio were showed at the IGF (Figure 1) and STG (Figure 2) respectively.

\section{- The safety and compliance}

The following measures were used to monitor the safety of acupuncture. The possible adverse events as well as acupuncture compliance were directly reported to the research team or via the Weekly Parental Report by parents; Acupuncturist and clinicians directly observed possible adverse events and compliance for each case during the acupuncture.

\section{- Statistical analysis}

The baseline characteristics and differences between acupuncture group and control group with different outcome measures (ATEC, PEDI, RDLS, GDDS/WPPSI-IV/WISC-IV, CGI-I,

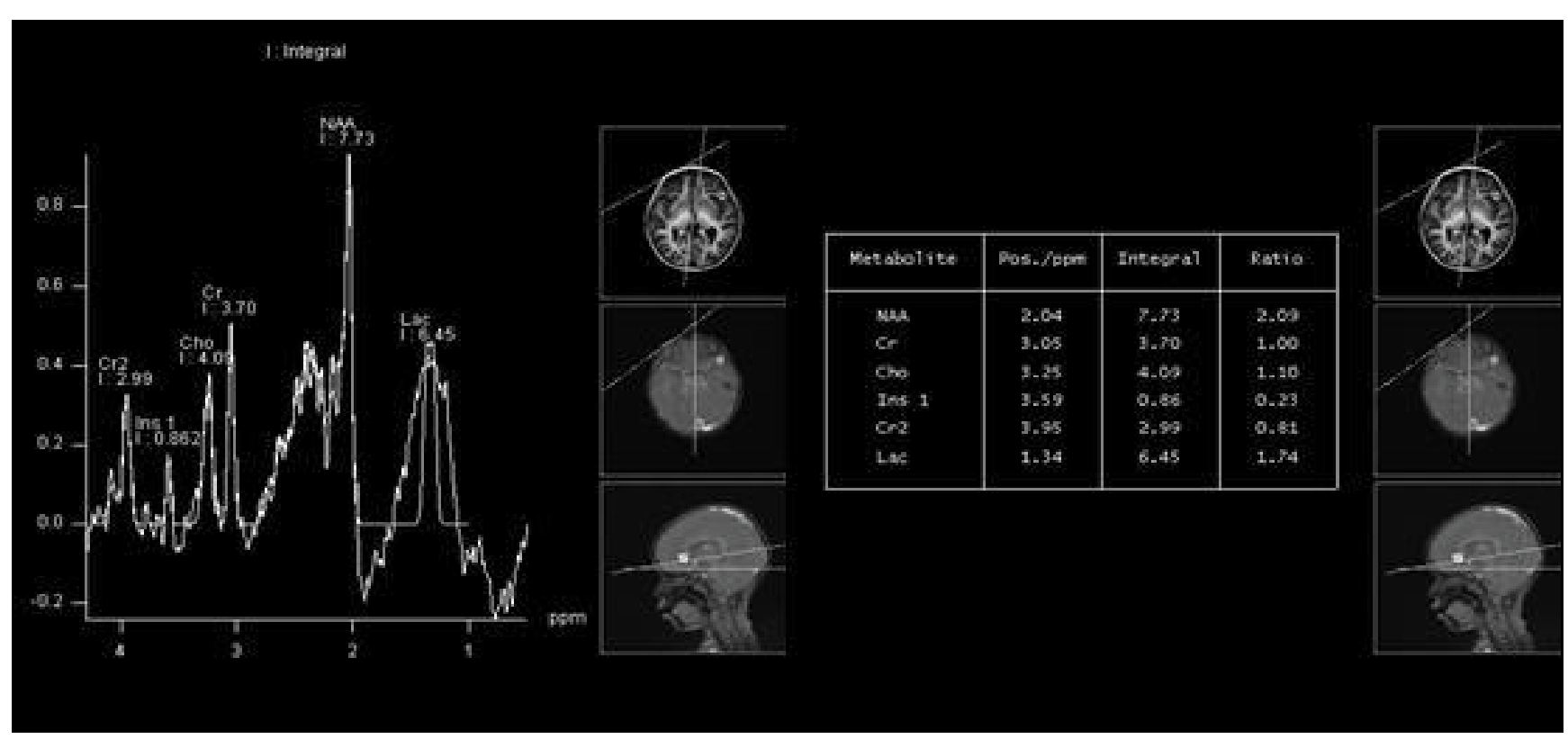

Figure 1: MRS line and compound ratio in the grey matter of left inferior frontal gyrus. 


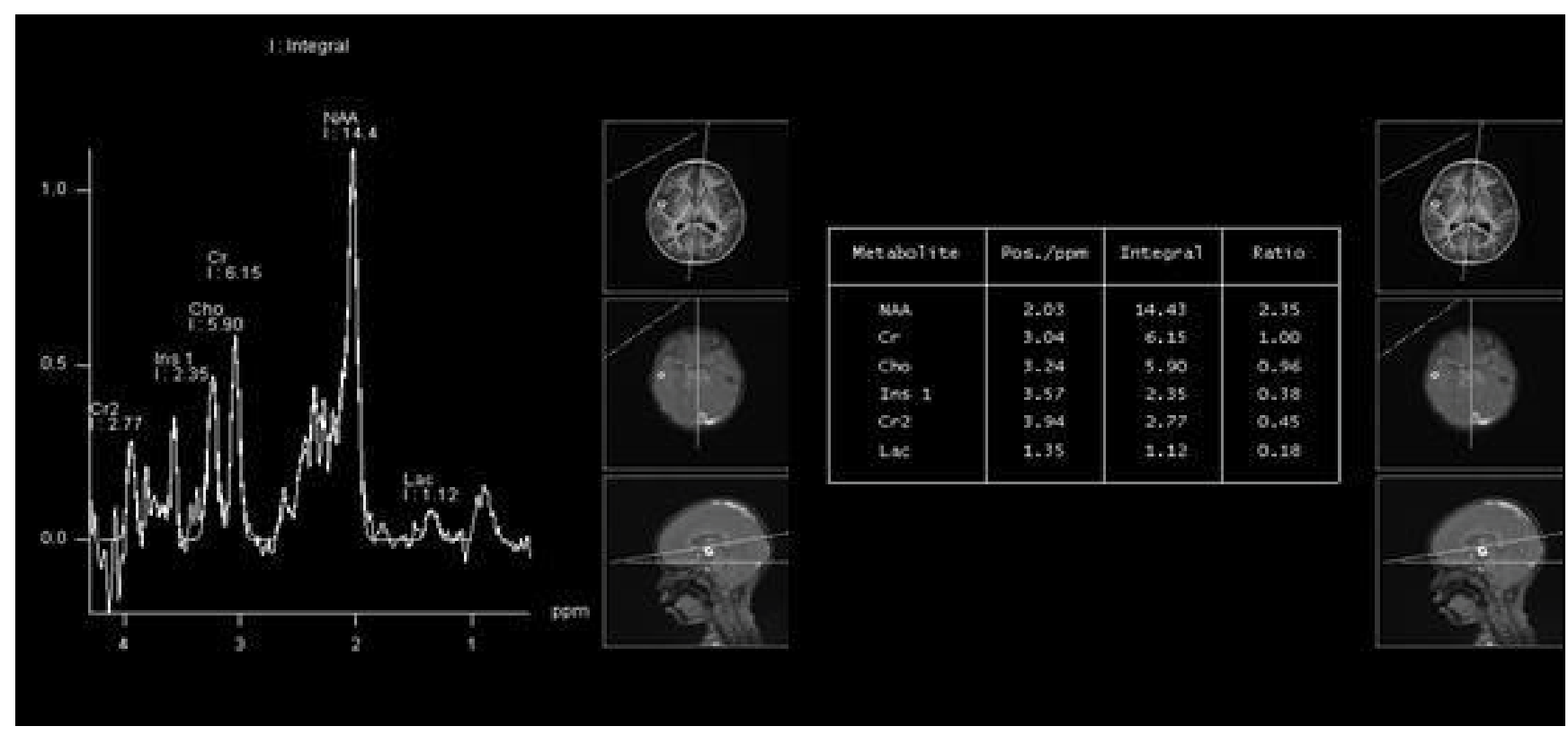

Figure 2: MRS line and compound ratio in the gray matter of right superior temporal gyrus.

Weekly Parental Report, MRS) were analyzed using the Mann-Whitney test. The intention-totreat approach was adopted, with the exception of MRS data. A significant difference is defined as Alpha $<0.05$.

\section{Results}

\section{- Baseline characteristics}

The enrollment of the flow chart of current RCT was showed in the Figure 3. Of a total of 66 responders was received. 47 subjects were enlisted and were randomly assigned to acupuncture (24 cases) or control group (23 cases). The four subjects defaulted after randomization, leaving 43 cases started the study- 24 cases in the acupuncture group and 19 cases in the control group.

Forty one subjects (23, acupuncture group; 18 , control group) were suitable for post-treatment evaluation because one subject from acupuncture group defaulted after 9 acupuncture sessions due to the fever with the acute upper respiratory tract infection, and another control case dropped out. 18 out of 24 cases in the acupuncture group and only 8 out of 19 cases in the control group received before and after MRS assessments. Those with the aged under 2 years old would be followed until 2 years old with the confirmed diagnosis.

The characteristics of baseline in both groups were shown in Table 1, including age, gender, birth weeks/birth weight, severity of autism, social status, parental educational status, acceptance of neuroleptic drugs, accepted acupuncture before. There were no significant differences between acupuncture and control group, regarding the baseline characteristics.

\section{- Outcome measures}

The differences of various outcome measures with ATEC, PEDI, and CGI-I by parents and RDLS, GDDS/WPPSI-IV/WISC-IV by assessor before and after acupuncture between both groups were summed up in the Table 2, which showed significant improvements on the self-care $(\mathrm{p}=0.045)$ and self-care assistant $(\mathrm{p}=0.019)$ domains of PEDI, and CGI-I scale $(\mathrm{p}=0.003)$ in the acupuncture group compared with the control group. The Table 3 also showed that the patients in the acupuncture group had significantly better social initiation $(\mathrm{P}=0.003)$, receptive language $(\mathrm{p}=0.002)$, pointing ( $\mathrm{p}=0.036)$, imitation $(\mathrm{p}=0.025)$, and motor skills $(\mathrm{p}=0.036)$, in terms of Weekly Parental Report.

As for the outcomes of MRS, the acupuncture group showed significantly decreased the ratio of the concentrations of Choline /Creatine $(p=0.037)$ at the grey matter of left inferior frontal gurus and increased the concentrations of Creatine $(p=0.036)$ at the grey matter of right superior temporal gurus (Table 4).

\section{- Compliance and side effects}

All subjects accomplished all acupuncture sessions, with the exception of one case 


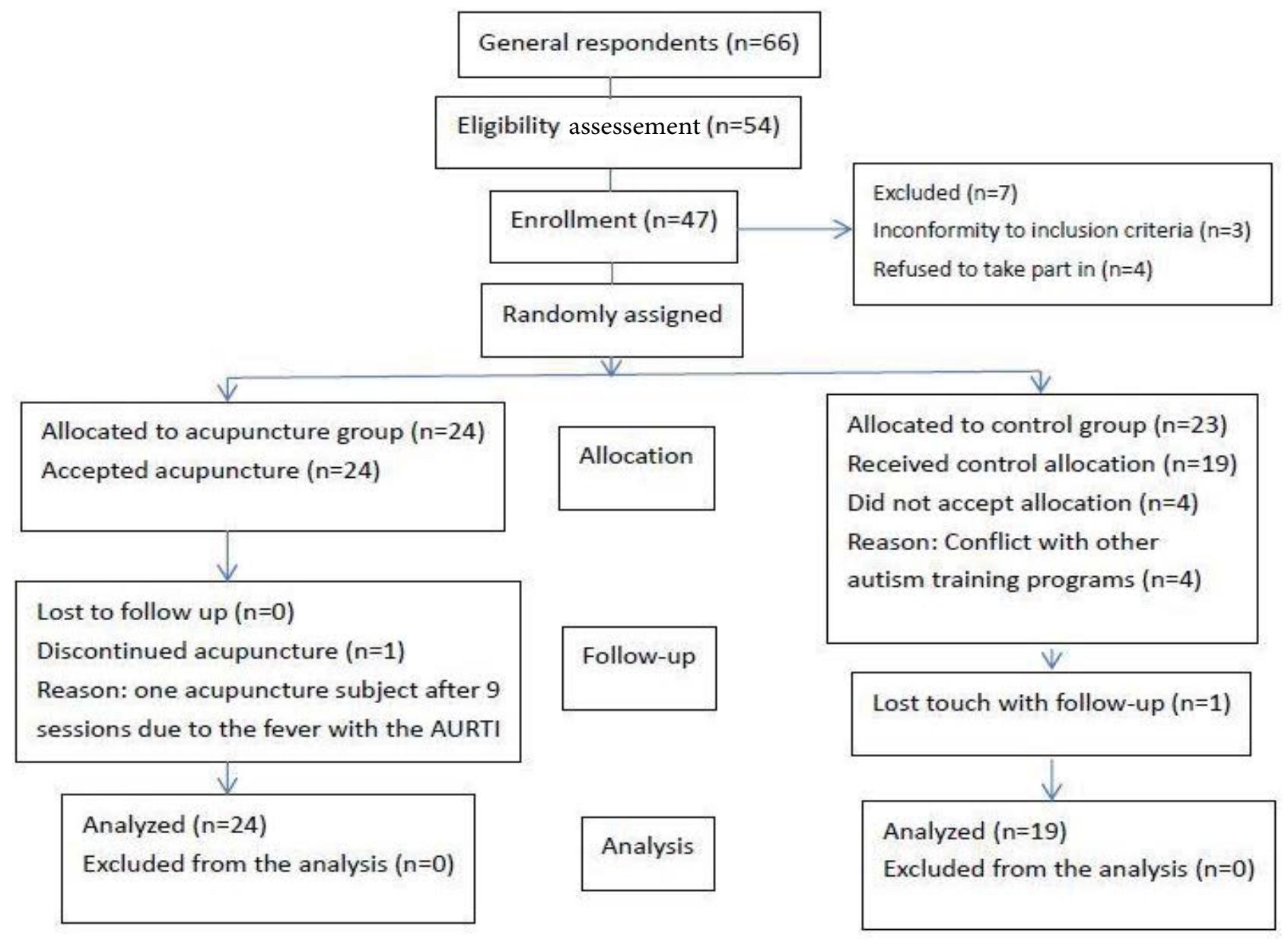

Figure 3: The enrollment flow chart.

AURTI: Acute Upper Respiratory Tract Infection

disconnected after 9 sessions due to the fever with the acute upper respiratory tract infection, although the subjects might be needed for gentle holding in their initial acupuncture sessions. Initial crying with irritability was observed in the initial few sessions for some subjects, however, most subjects (19 out of 24; 79.17\%) adapted easily within the three sessions and all cases tolerated the technique well within the nine sessions.

\section{Discussion}

It is proposed that ASD might be due to an interaction between genetic, environmental and immunological factors, with oxidative stress as a mechanism involvement with these risk factors [24]. Study has also paid attention to the role of synapse structure and its function as one of the pivots to the development of ASD and implies possible targets for interventions [25]. In addition, the controversy over whether there is a correlation between dietary iron levels and the incidence of autism has also aroused concern [26]. The proved effective in alleviating problems of impaired social interaction and delayed verbal communication, regarding various intervention methodologies such as acupuncture, was needed. Traditionally, acupuncture and related techniques have been suggested for the management of cancer pain[27], while acupuncture for low back pain is one of the most commonly used non-pharmacological painrelieving techniques is also stated [28].

Attention should be paid to the interventions for the children with non-verbal autism, since they almost belong to a neglected group (containing around $25 \%$ ) due to the following long-standing misundersatndings according to the traditional concepts: (1) older patients no longer respond to intervention; (2) older patients are no longer considered to be need to intervention, although a comprehensive review [29] has summarized 


\begin{tabular}{|c|c|c|c|c|c|c|c|}
\hline & \multicolumn{3}{|c|}{ Acupuncture group } & \multicolumn{3}{|c|}{ Control group } & \multirow[b]{2}{*}{ P value } \\
\hline & $\mathbf{N}$ & Mean & SD & $\mathbf{N}$ & Mean & SD & \\
\hline $\begin{array}{l}\text { Chronological } \\
\text { age (year) }\end{array}$ & 24 & 3.03 & 1.42 & 19 & 3.40 & 1.28 & 0.212 \\
\hline $\begin{array}{l}\text { Gender } \\
\text { (Male/female) }\end{array}$ & $20 / 4$ & & & $17 / 2$ & & & 0.568 \\
\hline Birth Weeks & 24 & 38.98 & 2.13 & 19 & 39.31 & 1.33 & 0.806 \\
\hline Birth Weight & 24 & 3159.58 & 699.57 & 19 & 3365.26 & 397.92 & 0.419 \\
\hline $\begin{array}{l}\text { Severityof } \\
\text { autism (CARS) }\end{array}$ & 24 & 35.65 & 3.23 & 19 & 35.24 & 3.53 & 0.484 \\
\hline \multicolumn{8}{|l|}{ Social status (parents) } \\
\hline Professional & 2 & & & 2 & & & 0.406 \\
\hline $\begin{array}{l}\text { Manegerical \& } \\
\text { Technical }\end{array}$ & 14 & & & 9 & & & \\
\hline $\begin{array}{l}\text { Clerical and minor supervisory } \\
\text { and } \\
\text { skilled manual }\end{array}$ & 18 & & & 11 & & & \\
\hline $\begin{array}{l}\text { Semi-skill } \\
\text { manual }\end{array}$ & 4 & & & 6 & & & \\
\hline $\begin{array}{l}\text { Unskilled } \\
\text { manual }\end{array}$ & 10 & & & 10 & & & \\
\hline \multicolumn{8}{|c|}{ Parent education status (Parental/Maternal education) } \\
\hline $\begin{array}{l}\text { University or } \\
\text { above }\end{array}$ & $27(15 / 12)$ & & & $20(11 / 9)$ & & & 0.660 \\
\hline Middle school & $19(8 / 11)$ & & & $15(6 / 9)$ & & & \\
\hline Primary school & $2(1 / 1)$ & & & $3(2 / 1)$ & & & \\
\hline Illiterate & $0(0 / 0)$ & & & $0(0 / 0)$ & & & \\
\hline \multicolumn{8}{|l|}{ Accepted acupuncture before } \\
\hline Yes & 0 & & & 0 & & & 1.000 \\
\hline No & 24 & & & 19 & & & \\
\hline \multicolumn{8}{|l|}{ Acceptance of neuroleptic drug } \\
\hline Yes & 0 & & & 0 & & & 1.000 \\
\hline No & 24 & & & 19 & & & \\
\hline \multicolumn{8}{|c|}{ CARS: Childhood Autism Rating Scale } \\
\hline
\end{tabular}

the literature since 1951-2008 and found that 167 autistic patients still acquired speech at or after the age of 5 years old. Secondly, ASD is a clinical phenotypic heterogeneous spectrum disorder, so that the subjects who are selected as homogeneously as possible may help researcher more easily to find the true effect on certain sub-group of ASD (e.g. non-verbal autism) via certain intervention (e.g. acupuncture). Furthermore, from the point of view of the individual dialectical treatment of traditional Chinese medicine (TCM), the inclusion of a group of the homogeneous (e.g.non-verbal autistic children) can help minimize the deviation of the dialectical treatment of TCM accordingly. Finally, the homogenization as much as possible allow researchers to explore some good potential [effective, economical, methodology of simple (e.g. current study only select two acupoints on head), and minor sideeffect] alternative therapies such as acupuncture, form a standardized treatment prescription for a subtype of ASD such as non-verbal autism, after via evidence-based medicine study, and maybe eventually being a standard treatment program for the benefit of a subtype of autistic patients.

The dysregulation of mirror neuron system (MNS) and its correlative networks are deemed to be associated with communication, social as well as emotional regulation of ASD [6]. Study has revealed that the performance of the mirror neurons can be changed by training [12].

Based on Traditional Chinese Medicine (TCM) philosophy, the health is achieved by holding non-stagnated flow of "Qi" along 14 meridians throughout the body. Disease is brought about by stagnation to the flow of this "Qi" or energy [30]. Acupuncture could help to regain the smooth flow of Qi, thus renewing the internal balance [30]. The TCM rationale for ASD is more holistic [31]. The pathogenesis of ASD is the derangement and defficiency of the Brain and Mind [31]. 
Research Dr.Wen-Xiong Chen

\begin{tabular}{|c|c|c|c|c|c|c|c|}
\hline & \multicolumn{3}{|c|}{ Acupuncture group } & \multicolumn{3}{|c|}{ Control group } & \multirow[b]{2}{*}{$\begin{array}{l}P \\
\text { value }\end{array}$} \\
\hline & $\mathbf{N}$ & Mean & SD & $\mathbf{N}$ & Mean & SD & \\
\hline \multicolumn{8}{|c|}{ Autism Treatment Evaluation Checklist (ATEC) } \\
\hline Speech/Language/Communication & 24 & -1.63 & 3.45 & 19 & -0.42 & 3.04 & 0.401 \\
\hline Sociability & 24 & -2.92 & 4.22 & 19 & -2.21 & 4.96 & 0.703 \\
\hline Sensory/Cognitive awareness & 24 & -1.17 & 4.33 & 19 & 0.16 & 5.16 & 0.531 \\
\hline Health/Physical/Behavior & 24 & -1.50 & 8.46 & 19 & -0.37 & 6.01 & 0.650 \\
\hline Total score & 24 & -7.92 & 13.29 & 19 & -4.00 & 11.92 & 0.372 \\
\hline \multicolumn{8}{|c|}{ Gesell Developmental Diagnostic Scale (GDDS)/WSIC-IV/WPPSI-IV } \\
\hline Developmental quotient (DQ)/IQ & 24 & 2.00 & 2.55 & 19 & 1.89 & 2.94 & 1.000 \\
\hline \multicolumn{8}{|c|}{ Pediatric Evaluation of Disability Inventory (PEDI) } \\
\hline Self-care & 24 & 4.00 & 4.32 & 19 & 0.79 & 5.53 & 0.045 \\
\hline Mobility & 24 & 0.92 & 1.86 & 19 & 0.53 & 2.93 & 0.592 \\
\hline Cognition & 24 & 1.96 & 4.54 & 19 & 2.05 & 4.14 & 0.931 \\
\hline Self-care caregiver assistant & 24 & 4.08 & 4.76 & 19 & 0.84 & 3.80 & 0.019 \\
\hline Mobility caregiver assistant & 24 & 2.17 & 3.41 & 19 & 0.47 & 3.03 & 0.177 \\
\hline Social caregiver assistant & 24 & 2.38 & 3.91 & 19 & 0.84 & 1.64 & 0.170 \\
\hline \multicolumn{8}{|c|}{ Reynell Developmental Language Scale (RDLS) } \\
\hline Comprehension (raw score) & 24 & 1.08 & 2.17 & 19 & 1.21 & 2.10 & 0.920 \\
\hline Production (raw score) & 24 & 0.17 & 0.64 & 19 & 0.21 & 0.54 & 0.482 \\
\hline \multicolumn{8}{|c|}{ Clinical Global Impression-Improvement (CGI-I) scale } \\
\hline Much improvement & 6 & & & 1 & & & 0.003 \\
\hline Minimal improvement & 16 & & & 9 & & & \\
\hline No change & 2 & & & 9 & & & \\
\hline
\end{tabular}

One randomized controlled trial (RCT) showed that a short course of electro-acupuncture was helpful to ameliorate specific functions such as language comprehension and self-care ability in children with ASD [32], while another RCT reported that the scalp acupuncture being a safe complementary modality had a significant effect on language development in autistic children, in together with the language therapy [33]. In addition, more recent study reported that the scalp acupuncture could ameliorate verbal communication problems the most while noise sensitivity improved the least. The therapeutic effectiveness decreased along with the age of growth and children with natal autism benefit more from acupuncture than those with regressive autism [34].

According to the philosophy of TCM, the selected two acupoints in current RCT are situated in the Hand Foot Shaoyang meridian [13]. The rationale of acupuncture for selected acupoints was to burgeon Qi and dredged up Qi of the liver and bile meridian, being beneficial to brain function accordingly [13], holding the characteristics of germinal life and growth in nature [13]. A close relationship between the nervous system and the acupoint is stated [35]. Presumably, an anatomical close relationship between the selected acupoints [the temporal anterior oblique (MS6) under 2/5 and temporal posterior oblique (MS7) under 2/5] and the jacent center nervous systems including the Broca area and superior temporal sulcus (both containing MNS) [13] might existed. As a result, acupuncture might be involvement in the MNS indirectly, and consequently ameliorated the social relatedness, communication and related symptoms of non-verbal autistic children, via regulating the dysfunctional MNS accordingly [13].

Research has showed the relationship between joint attention and language development in autistic children. A significant improvement of the "pointing" domain of Weekly Parental Report in the current RCT was reported $(\mathrm{p}=0.036)$ (Table 4), in accordance with the amelioration of joint attention in some cases in our previous pilot study [13], e.g. Case 3 pointing to moon for her mother. Many ASD subjects being lack of imitation abilities were revealed. The significant improvements $(\mathrm{p}=0.025)$ of the imitation ability were also found in our RCT in terms of Weekly Parental Report, in consistent with the outcomes of our pilot study [13], e.g. Case 1 listening to the phone, using a cotton ball to wipe the skin. Furthermore, in those autistic children with praxic deficits, the quality and quantity of the imitation ability are also impaired, with 


\section{Acupuncture for Non-Verbal Autistic Children: Randomized Controlled Trial Research}

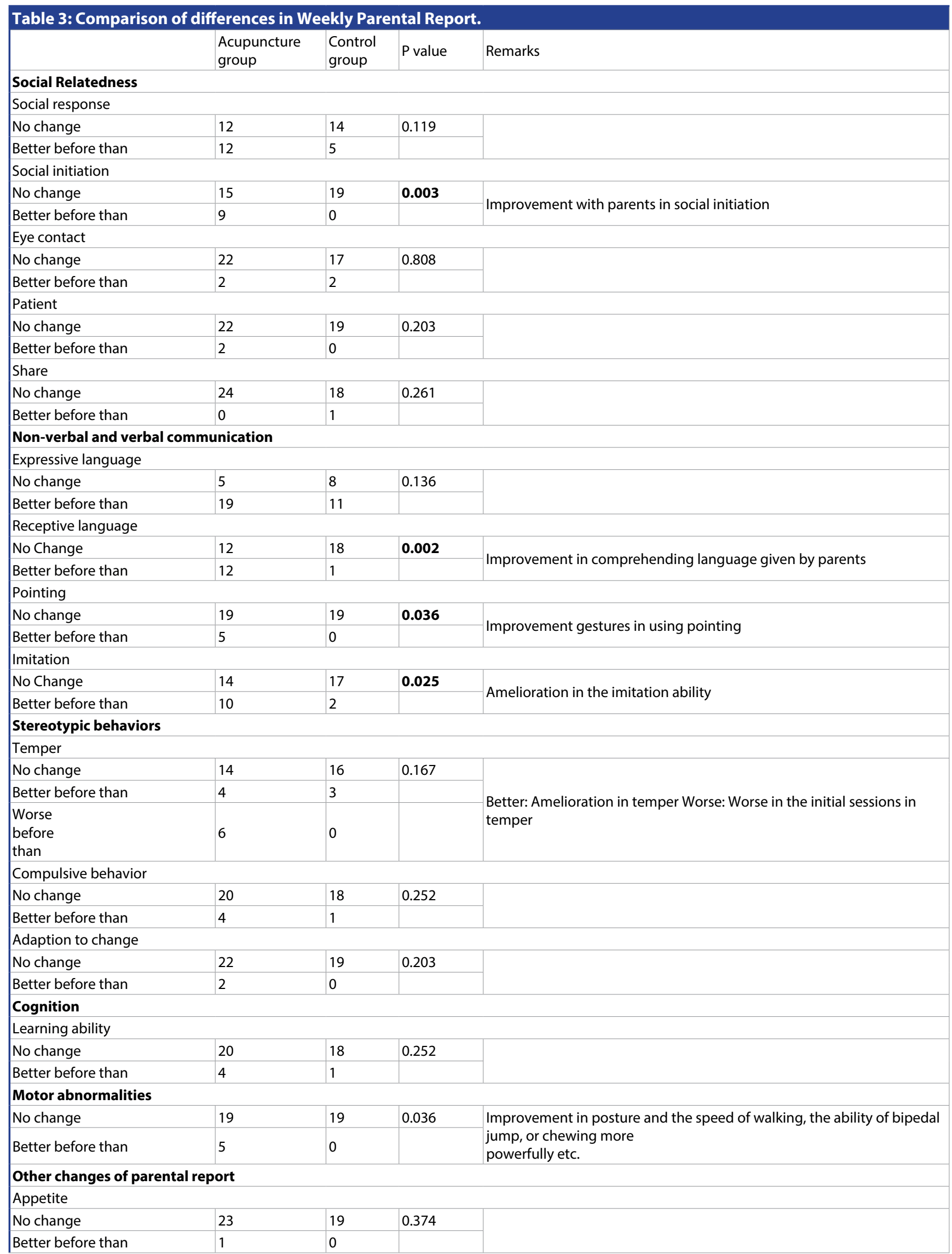


Research Dr.Wen-Xiong Chen

\begin{tabular}{|c|c|c|c|c|}
\hline \multicolumn{5}{|l|}{ Sleeping pattern } \\
\hline No change & 19 & 18 & 0.167 & \multirow{3}{*}{$\begin{array}{l}\text { Better: amelioration in sleep pattern; Worse: worse in sleeping pattern in } \\
\text { the initial sessions (wake earlier or sleep later) }\end{array}$} \\
\hline Better before than & 3 & 0 & & \\
\hline $\begin{array}{l}\text { Worse } \\
\text { before } \\
\text { than }\end{array}$ & 2 & 1 & & \\
\hline \multicolumn{5}{|l|}{ Attention span } \\
\hline No change & 23 & 18 & 0.867 & \\
\hline \multirow[t]{2}{*}{ Better before than } & 1 & 1 & & \\
\hline & $\begin{array}{l}\text { Acupuncture } \\
\text { group }\end{array}$ & $\begin{array}{l}\text { Control } \\
\text { group }\end{array}$ & $P$ value & Remarks \\
\hline \multicolumn{5}{|l|}{ Social Relatedness } \\
\hline \multicolumn{5}{|l|}{ Social response } \\
\hline No change & 12 & 14 & 0.119 & \\
\hline Better before than & 12 & 5 & & \\
\hline \multicolumn{5}{|l|}{ Social initiation } \\
\hline No change & 15 & 19 & 0.003 & \multirow{2}{*}{ Improvement with parents in social initiation } \\
\hline Better before than & 9 & 0 & & \\
\hline \multicolumn{5}{|l|}{ Eye contact } \\
\hline No change & 22 & 17 & 0.808 & \\
\hline Better before than & 2 & 2 & & \\
\hline \multicolumn{5}{|l|}{ Patient } \\
\hline No change & 22 & 19 & 0.203 & \\
\hline Better before than & 2 & 0 & & \\
\hline \multicolumn{5}{|l|}{ Share } \\
\hline No change & 24 & 18 & 0.261 & \\
\hline Better before than & 0 & 1 & & \\
\hline \multicolumn{5}{|c|}{ Non-verbal and verbal communication } \\
\hline \multicolumn{5}{|c|}{ Expressive language } \\
\hline No change & 5 & 8 & 0.136 & \\
\hline Better before than & 19 & 11 & & \\
\hline \multicolumn{5}{|l|}{ Receptive language } \\
\hline No Change & 12 & 18 & 0.002 & \multirow{2}{*}{ Improvement in comprehending language given by parents } \\
\hline Better before than & 12 & 1 & & \\
\hline \multicolumn{5}{|l|}{ Pointing } \\
\hline No change & 19 & 19 & 0.036 & \multirow{2}{*}{$\begin{array}{l}\text { Improvement gestures in } \\
\text { Using pointing }\end{array}$} \\
\hline Better before than & 5 & 0 & & \\
\hline \multicolumn{5}{|l|}{ Imitation } \\
\hline No Change & 14 & 17 & 0.025 & \multirow{2}{*}{ Amelioration in the imitation ability } \\
\hline Better before than & 10 & 2 & & \\
\hline \multicolumn{5}{|c|}{ Stereotypic behaviors } \\
\hline Temper & & & & \\
\hline No change & 14 & 16 & 0.167 & \\
\hline Better before than & 4 & 3 & & $\begin{array}{l}\text { Better: Amelloration in temper worse: Worse in the initial sessions in } \\
\text { temper }\end{array}$ \\
\hline Worse before than & 6 & 0 & & \\
\hline Compulsive behavic & & & & \\
\hline No change & 20 & 18 & 0.252 & \\
\hline Better before than & 4 & 1 & & \\
\hline Adaption to change & & & & \\
\hline No change & 22 & 19 & 0.203 & \\
\hline Better before than & 2 & 0 & & \\
\hline Cognition & & & & \\
\hline Learning ability & & & & \\
\hline No change & 20 & 18 & 0.252 & \\
\hline Better before than & 4 & 1 & & \\
\hline Motor abnormaliti & & & & \\
\hline
\end{tabular}




\begin{tabular}{|c|c|c|c|c|}
\hline No change & 19 & 19 & 0.036 & \multirow{2}{*}{$\begin{array}{l}\text { Improvement in posture and the speed of walking, the ability of bipedal } \\
\text { jump, or chewing more } \\
\text { powerfully etc. }\end{array}$} \\
\hline Better before than & 5 & 0 & & \\
\hline \multicolumn{5}{|c|}{ Other changes of parental report } \\
\hline \multicolumn{5}{|l|}{ Appetite } \\
\hline No change & 23 & 19 & 0.374 & \\
\hline Better before than & 1 & 0 & & \\
\hline \multicolumn{5}{|l|}{ Sleeping pattern } \\
\hline No change & 19 & 18 & 0.167 & \multirow{3}{*}{$\begin{array}{l}\text { Better: amelioration in sleep pattern; Worse: worse in sleeping pattern in } \\
\text { the initial sessions (wake earlier or sleep later) }\end{array}$} \\
\hline Better before than & 3 & 0 & & \\
\hline Worse before than & 2 & 1 & & \\
\hline \multicolumn{5}{|l|}{ Attention span } \\
\hline No change & 23 & 18 & 0.867 & \\
\hline Better before than & 1 & 1 & & \\
\hline
\end{tabular}

\begin{tabular}{|c|c|c|c|c|c|c|c|}
\hline & \multicolumn{3}{|c|}{ Acupuncture group } & \multicolumn{3}{|c|}{ Control group } & \multirow[b]{2}{*}{$\begin{array}{l}P \\
\text { value }\end{array}$} \\
\hline & $\mathbf{N}$ & Mean & SD & $\mathbf{N}$ & Mean & SD & \\
\hline \multicolumn{8}{|l|}{ Basic characteristics } \\
\hline Chronological age (year) & 18 & 3.21 & 1.58 & 8 & 3.98 & 1.24 & 0.129 \\
\hline Gender (Male/Female) & $16 / 2$ & & & $8 / 0$ & & & 0.336 \\
\hline Severity of autism (CARS) & 18 & 35.94 & 3.49 & 8 & 36.81 & 4.49 & 0.780 \\
\hline $\mathrm{DQ} / \mathrm{IQ}$ & 18 & 50.61 & 12.21 & 8 & 44.13 & 12.35 & 0.173 \\
\hline \multicolumn{8}{|c|}{ Right frontal inferior gurus (grey matter) } \\
\hline R FIGGNAA & 18 & -0.75 & 5.75 & 8 & -0.95 & 4.35 & 0.739 \\
\hline R FIGGNAA/CR & 18 & -0.24 & 1.35 & 8 & 0.08 & 1.01 & 0.617 \\
\hline R FIGGCR & 18 & -0.08 & 1.78 & 8 & -0.51 & 1.26 & 0.541 \\
\hline R FIGGCHO & 18 & 0.63 & 2.61 & 8 & -0.26 & 1.62 & 0.470 \\
\hline R FIGGCHO/CR & 18 & 0.84 & 0.29 & 8 & 0.06 & 0.12 & 0.956 \\
\hline \multicolumn{8}{|c|}{ Left frontal inferior gurus (grey matter) } \\
\hline L FIGGNAA & 18 & -0.85 & 3.57 & 8 & -2.94 & 4.92 & 0.294 \\
\hline L FIGGNAA/CR & 18 & -0.25 & 0.43 & 8 & -0.27 & 0.83 & 0.977 \\
\hline L FIGGCR & 18 & 0.17 & 1.36 & 8 & -0.87 & 2.50 & 0.268 \\
\hline L FIGGCHO & 18 & 0.22 & 1.61 & 8 & 0.09 & 2.92 & 0.907 \\
\hline L FIGGCHO/CR & 18 & -0.00 & 0.16 & 8 & 0.17 & 0.19 & 0.036 \\
\hline \multicolumn{8}{|c|}{ Right temporal superior gurus (grey matter) } \\
\hline R TSGGNAA & 18 & 0.38 & 3.37 & 8 & -1.10 & 2.56 & 0.201 \\
\hline R TSGGNAA/CR & 18 & -0.31 & 0.56 & 8 & -0.04 & 0.50 & 0.211 \\
\hline R TSGGCR & 18 & 0.80 & 1.39 & 8 & -0.39 & 1.19 & 0.037 \\
\hline R TSGGCHO & 18 & 0.92 & 2.05 & 8 & 0.40 & 1.77 & 0.359 \\
\hline R TSGGCHO/CR & 18 & -0.02 & 0.16 & 8 & 0.07 & 0.13 & 0.290 \\
\hline \multicolumn{8}{|c|}{ Left temporal superior gurus (grey matter) } \\
\hline L TSGGNAA & 18 & -0.64 & 4.25 & 8 & -0.49 & 4.27 & 0.956 \\
\hline L TSGGNAA/CR & 18 & -0.13 & 0.52 & 8 & -0.16 & 0.80 & 0.541 \\
\hline L TSGGCR & 18 & -0.04 & 1.92 & 8 & 0.04 & 1.54 & 0.934 \\
\hline L TSGGCHO & 18 & -0.12 & 1.68 & 8 & 0.13 & 1.55 & 0.739 \\
\hline L TSGGCHO/CR & 18 & -0.02 & 013 & 8 & 0.02 & 0.12 & 0.487 \\
\hline
\end{tabular}

the locomotion as awkward and inaccurate. Interestingly, significant improvements in motor skills (Table 3; $\mathrm{p}=0.036$ ) was also found in the acupuncture group.

The occurrence of cognitive impairments is also supposed to play important roles in the poor language acquisition [2]. There was significant improvement in the developmental quotient (DQ) $(\mathrm{p}=0.018)$ of cases in a small case series [13], although no significant improvements on the DQ/IQ assessments after acupuncture of current RCT were found.

The improvements of social initiation $(\mathrm{p}=0.003)$ and receptive language $(\mathrm{p}=0.002)$ were also 
stated based on the outcomes of Weekly Parental Report, although no significant changes in the domain of Speech/Language/Communication and sociability of ATEC and the outcomes of RDLS either in Comprehension or Production domain were found.

There were not significant improved in the stereotyped behaviors in current RCT according to the outcomes of Weekly Parental Report (Table 3), although the outcomes of our pilot study [14] showed less compulsive behaviors in some cases, such as Case 1 with no longer persistently played ball and reduced the "flicking" action.

Regarding the functional status of outcome measures, the significant improvement in the self-care (Table 2; $\mathrm{p}=0.045$ ) and self-care assistant (Table 2; $\mathrm{p}=0.019$ ) domains of PEDI were found.

The amelioration of the self-care, self-care assistant, and other domains may be conductive to the significant amelioration of the clinical global impression of CGI-I (Table 2; $\mathrm{p}=0.003$ ).

Autistic children may come through side effects of acupuncture but can not convey relevant information to their parents or the researchers appropriately because of the communication impairment [31]. The definition of "acupuncture compliance" is that subjects can sit or lie on a couch to accept acupuncture, although they cry or needed gentle hand or head holding [32]. Good compliance was defined as being able to finish acupuncture within the initial three sessions [32]. All cases in current RCT received acupuncture first time and most possessed good compliance.

A systematic reviews of the acupuncture safety revealed that the most common side effects were needle pain, tiredness, and bleeding, with the uncommon of feeling of the faintness and syncope, and rare event of pneumothorax [36]. In current study, the crying and irritability as mild side effects at the initial sessions during acupuncture were underwent by some subjects, although worse sleeping pattern or temper during a couple of initial sessions of a few patients were also reported in current RCT and previous study [32], based on the Parental Report, warranting the further study in the future relevant acupuncture studies.

We also explored the changes of brain neurochemical after acupuncture, via modern neuroimaging technique. The fact that autism is a neurobiological disorder has been widely accepted, although specific biological markers have not been discovered as yet. Proton MR Spectroscopy (MRS) allows for evaluation of various chemical metabolites in non-invasive manner. Therefore, Current RCT used Proton MRS to study the effects of acupuncture on the metabolism of neurochemical at the inferior frontal gyrus and at the superior temporal gyrus respectively, which both area consist of MNS, and tried to illustrate the possible mechanism of acupuncture for non-verbal autistic children.

Most prone MRS studies in ASD have been performed in children and have revealed general and localized reduction in $\mathrm{N}$-acetylaspartate (NAA) concentration [37,38]. NAA concentration is thought to be a measure of neural density and mitochondrial function [39].

Choline-containing complexes (Cho), including phosphocholine and choline, are one of the components of cell membrane phospholipid metabolism, reflecting changes in cell membrane and markers of cell renewal. Reductions in Cho, considered a measure of phosphate membrane turnover, have also been observed in the temporal lobe, the anterior cingulate cortex (ACC), and thalamus, while increased Cho concentrations have been reported in the frontal lobes and head of the caudate nucleus in patients with ASD [40, 41]. A study [42] showed that the ratio of $\mathrm{Cho} / \mathrm{Cr}$ in the amygdala of the left hippocampus was significantly higher in the speech impaired ASD group, compared with the non-verbal impairment ASD group and control group. Sokol et al. [43] also reported that increased ratio of $\mathrm{Cho} / \mathrm{Cr}$ in temporal lobe, was associated with severity of autism symptoms. Interestingly, in contrast to control group, the acupuncture group in current RCT showed significantly decreased the ratio of the concentrations of Choline /Creatine $(p=0.037)$ at the left inferior frontal gurus.

Creatine $(\mathrm{Cr})$ and phosphocreatine levels are a reflection of cellular adenosine triphosphate (ATP) metabolism [44], and play an essential role in ATP and adenosine diphosphate (ADP) energy transfer and equilibrium within cells. $\mathrm{Cr}$ and $\mathrm{Cr}$ kinase are essential in cellular energy metabolism and in the maintenance of cortical homeostasis [45]. Studies showed that $\mathrm{Cr}$ reduction across the cortex in children with ASD compared with control groups [37,40,41,46,47]. Turner and Gant [48] provided an extensive review of the biochemistry of creatine, reporting 
an association between reduced $\mathrm{Cr}$ and abnormal speech and motor learning, intellectual disability and ASD-like behaviors. It is suggested that these are symptoms of delayed or impaired axon growth during development, of which $\mathrm{Cr}$ is an important in gradient [48]. Our study revealed the increased the concentrations of Creatine $(p=0.036)$ at the right superior gurus after acupuncture.

Although the improvements in the functional domains (self-care) and some core features (social initiative and receptive language) of non-verbal autistic children were revealed in current RCT study, there were some limitations as follows. First, this RCT is a limited numbers including only 24 cases in the acupuncture group and 19 cases in the control group; small numbers of only 8 patients in the control group finishing the MRS study. Secondly, the symptoms of young subjects in the study might improve automatically even in the short time period (4 weeks) due to the developmental processes in nature. Thirdly, except for the Self-care domains, the improvement of social initiative and other related domains were mostly supported by Weekly Parental Report, rather than the standard assessment scales such as PEDI.

In sum, the short term extensive acupuncture for selected acupoints might regulate the MNS cellular renewal as well as cellular energy metabolism, which may improve dysfunction of MNS and its related networks, leading to the improvements of communications (receptive language) and social relatedness (social initiation, imitation, pointing), and finally ameliorating the functional status (self-care) of non-verbal autistic children.

\section{Acknowledgments \\ We would like to thank all of the participants and their parents/guardians who participated in this study.}

\section{Funding source}

This research is supported by the grants of Science and Technology Department of Guangdong Province of China (2013B021800046 \& 2016A020215019) and Doctoral Fund of Guangzhou Women and Children's Medical Center (5001-2170057), and partly supported by the key projects fund of Ministry of science and technology of China (2016YFC1306200).

\section{Conflict of Interest}

There are no conflicts of interest for all authors in this study.

\section{Author contributions \\ CWX and LG conceptualized and designed the study. HG performed acupuncture. CWX, HZF, $Y S Y$ and HJY recruited and assessed the subjects. LHS, ZSH and WQQ performed MRS. CWX analyzed the data and drafted the manuscript. All authors have agreed on the final version.}

\section{References}

1. American Psychiatric Association. Diagnostic and Statistical Manual of Mental Disorders, $5^{\text {th }}$ Edtn. Arlington (VA): American Psychiatric Publishing (2013).

2. Venter A, Lord C, Schopler E. A follow-up study of high-functioning autistic children. J. Child. Psychol. Psychiatry 33(3), 489507(1992).

3. Volkmar FR, Lord C, Bailey A, et al. Autism and pervasive developmental disorders. J. Child. Psychol. Psychiatry 45(1),135-170 (2004).

4. Rutter M, Schopler E. Autism Diagnosis and definition: a reappraisal of concepts and treatment. New York: Plenum Press 1-25 (1978).

5. Rogers SJ, Hayden D, Hepburn S, et al. Teaching young nonverbal children with autism useful speech: A pilot study of the Denver model and PROMPT interventions. J. Autism. Dev. Disord 36(8), 1007-1024
(2006).

6. Baron-Cohen S, Leslie AM, Frith U. Does the autistic-child have a theory of mind?. Cognition 21(1), 37-46 (1985).

7. Barsalou LW. Perceptual symbol systems. Behav. Brain. Sci 22(4), 577-609 (1999).

8. Gangitano M, Mottaghy FM, PascualLeone A. Phase-specific modulation of cortical motor output during movement observation. Neuroreport 12(7), 1489-1492 (2001).

9. Baumann S, Koeneke S, Schmidt CF, et al. A network for audio-motor coordination in skilled pianists and non-musicians. Brain. Res 1161(1), 65-78 (2007).

10. Buccino G, Lui F, Canessa N, et al. Neura circuits involved in the recognition of actions performed by nonconspecifics: an fMRI study. J. Cogn. Neurosci 16(1), 114-126 (2004).

11. Cheuk DKL, Wong V, Chen WX.
Acupuncture for autism spectrum disorders (ASD). Cochrane. Database. Syst. Rev (9), CD007849 (2011).

12. Wan CY, Demaine K, Zipse L, et al. From music making to speaking: Engaging the mirror neuron system in autism. Brain. Res. Bull 82(3-4), 161-168 (2010).

13. Chen WX, Liu G, Liu HS, et al. Acupuncture for non-verbal autistic children: a small case series. Neuropsychiatry 7(5),557-566 (2017).

14. Lord C, Rutter M, Le Couteur A. Autism Diagnostic Interview-Revised: A revised version of a diagnostic interview for caregivers of individuals with possible pervasive developmental disorders. J. Autism. Dev. Disord 24(5), 659-685 (1994).

15. Lord C, Rutter M, DiLavore PC. Autism Diagnostic Observation Scale-Generic. Chicago: University of Chicago Press (1997).

16. Schopler E, Reichler RJ, Renner BR. The Childhood Autism Rating Scale (CARS) for Diagnostic Screening and Classification 
in Autism. New York: Irvington Publishers (1986).

17. Rimland B, Edelson M. Autism Treatment Evaluation Checklist. SanDiego:Autism Research Institute (1999).

18. Haley SM, Coster WJ, Ludlow LH, et al. Pediatric Evaluation of Disability Inventory: Development, standardization, and administration manual, Version 1.0. Boston MA: PEDI Research group (1992).

19. No authors listed. Rating scales and assessment instruments for use in pediatric psychopharmacology research. Psychopharmacol. Bull 21(4), 714-1124 (1985).

20. Gesell A, Amatruda CS, Knobloch H, et al. Gesell and Amatruda's Developmental Diagnosis: the Evaluation and Management of Normal and Abnormal Neuropsychologic Development in Infancy and Early Childhood. New York: Harper \& Row (1974).

21. Wechsler D. The Wechsler Preschool and Primary Scale of Intelligence-Fourth edition. London: Pearson (2003).

22. Wechsler D. The Wechsler intelligence scale for children-Fourth edition. London: Pearson (2003).

23. Reynell JK, Gruber CP. Reynell Developmental Language Scale. Los Angeles: Western Psychological Services (1990).

24. Chauhan A, Chauhan V. Oxidative stress in autism. Pathophysiology 13(3), 171-181 (2006).

25. Rapin I, Tuchman RF. What is new in autism?. Curr. Opin. Neurol 21(2), 143-149 (2008).

26. Padhye $U$. Excess dietary iron is the root cause for increase in childhood autism and allergies. Med. Hypoth 61(2), 220-222 (2003).

27. Lu WD, Rosenthal DS. Acupuncture for cancer pain and related symptoms. Curr. pain. Headache. Rep 17(3), 321 (2013).
28. Lim TK, Ma Y, Berger F, et al. Acupuncture and neural mechanism in the management of low back pain-an update. Medicines. (Basel) 5(3), E63 (2018).

29. Pickett E, Pullara O, Grady JO, et al. Speech acquisition in older nonverbal individuals with autism. Cog. Behav. Neurol 22(1), 1-21 (2009).

30. Wong V, Chen WX. Is acupuncture useful for cerebral palsy? What evidence do we have?: Trends in cerebral palsy research. Nova Science Publishers Inc. New York 139-165 (2005).

31. Chen WX, Wong V, Liu WL. Electroacupuncture for children with autism spectrum disorder: Pilot study of 2 cases. J. Altern. Complement. Med 14(8), 1057-1065 (2008).

32. Wong V, Chen WX, Liu WL. Randomized Controlled Trial of electro-acupuncture for autism spectrum disorder. Alter. Med. Rev 15(2),136-146 (2010).

33. Allam H, Eldine NG, Helmy G. Scalp acupuncture effect on language development in children with autism: a pilot study. J. Altern. Complement. Med 14(2),109-114 (2008).

34. Yau CH, Ip CL, Chau YY. The therapeutic effect of scalp acupuncture on natal autism and regressive autism. Chin. Med 13(1), 30 (2018).

35. Fu H. What is the material base of acupuncture? The nerves! Med. hypotheses 54(3), 358-359 (2000)

36. Ernst $E$, White AR. Prospective studies of the safety of acupuncture: a systematic review. Am. J. Med 110(6), 481-485 (2001).

37. DeVito TJ, Drost DJ, Neufeld RW, et al. Evidence for cortical dysfunction in autism: a proton magnetic resonance spectroscopic imaging study. Biol. Psychiatry 61(4), 465473 (2007).

38. Endo T, Shioiri T, Kitamura H, et al. Altered chemical metabolites in the amygdala- hippocampus region contribute to autistic symptoms of autism spectrum disorders. Biol. Psychiatry 62(9), 1030-1037 (2007).

39. Clark JB. N-acetyl aspartate: a marker for neuronal loss or mitochondrial dysfunction. Dev. Neurosci 20(4-5), 271-276 (1998).

40. Friedman SD, Shaw DW, Artru AA, et al Regional brain chemical alterations in young children with autism spectrum Disorder. Neurology 60(1), 100-107 (2003).

41. Levitt JG, O'Neill J, Blanton RE, et al. Proton magnetic resonance spectroscopic imaging of the brain in childhood autism. Biol. Psychiatry 54(12), 1355-1366 (2003).

42. Gibis L, Wei $\mathrm{H}$, Azizian $\mathrm{A}$, et al. 1H-magnetic resonance spectroscopy markers of cognitive and language ability in clinical subtypes of autism spectrum disorders. $J$. Child. Neurol 23(7), 766-774 (2008).

43. Sokol DK, Dunn DW, Edwards-Brown M, et al. Hydrogen proton magnetic resonance spectroscopy in autism: Preliminary evidence of elevated choline/creatine ratio. J. Child. Neurol 17(4), 245-249 (2002).

44. Rae CD. A guide to the metabolic pathways and function of metabolites observed in human brain $1 \mathrm{H}$ magnetic resonance spectra. Neurochem. Res 39(1), 1-36 (2014).

45. Ross B, Bluml S. Magnetic resonance spectroscopy of the human brain. Anat. Rec 265(2), 54-84 (2001).

46. Hardan AY, Minshew NJ, Melhem NM, et al. An MRI and proton spectroscopy study of the thalamus in children with autism. Psychiatry. Res 163(2), 97-105 (2008).

47. Corrigan NM, Shaw DW, Estes AM, et al. Atypical developmental patterns of brain chemistry in children with autism spectrum disorder. JAMA. Psychiatry 70(9), 964-974 (2013).

48. Turner CE, Gant N. The biochemistry of creatine. In Rothmanpages CS. Magnetic Resonance Spectroscopy. CA: Academic Press, San Diego, California 91-103 (2014). 